\title{
Parasitisme gastro-intestinal chez les animaux du parc zoologique de Hann au Sénégal
}

\author{
Laibané Dieudonné Dahourou ${ }^{1} *$ Oubri Bassa Gbati ${ }^{1}$ \\ Issaka Nacanabo ${ }^{1}$ Cheikhouna Diatta ${ }^{1}$ Louis Joseph Pangui ${ }^{1}$
}

\section{Mots-clés}

Primate, Carnivora, endoparasite, animal de zoo, Dakar, Sénégal

Submitted: 17 October 2016

Accepted: 21 June 2017

Published: 20 September 2017

DOI: $10.19182 /$ remvt.31389

\begin{abstract}
Résumé
Des échantillons de matières fécales ont été prélevés sur 24 animaux appartenant à huit espèces différentes du parc zoologique de Hann au Sénégal. Ils ont été analysés par la technique de coproscopie microscopique qualitative de Telemann-Rivas et celle quantitative en cellules de Mac Master. Sur l'ensemble des échantillons, 66,7\% étaient parasités et chaque animal positif était infesté par au moins une espèce d'œufs d'helminthes, alors que les protozoaires n'étaient présents que chez quatre animaux. Chez les carnivores, les œufs de parasites, comme Toxascaris leonina et Toxocara cati, et ceux d'ankylostomidés étaient les plus présents, alors que chez les primates les œufs de Trichuris sp. et Entamoeba sp. ont été identifiés. Cette étude constitue une base pour la mise en place de traitements chez ces animaux.
\end{abstract}

- Pour citer cet article : Dahourou L.D., Gbati O.B., Nacanabo I., Diatta C., Pangui L.J., 2017. Gastrointestinal parasitism in wildlife at Hann Zoological Park in Senegal. Rev. Elev. Med. Vet. Pays Trop., 70 (1): 25-28, doi: 10.19182/remvt.31389

\section{INTRODUCTION}

Face à la crise de la biodiversité à l'échelle planétaire et particulièrement africaine (Mooney, 2010), les zoos peuvent constituer des lieux indispensables à la conservation des espèces animales sauvages. Cependant, la captivité peut soumettre les animaux à des stress variés et importants. Selon Morgan et Tromborg (2007), l'absence ou la présence insuffisante de stimuli sensoriels, le confinement, le contact fréquent avec les humains, la mauvaise organisation sociale en captivité constituent des facteurs qui génèrent du stress. Or, ce dernier peut induire une réduction de la fonction immunitaire (Asres et Amha, 2014) avec comme conséquence l'apparition de maladies chez les animaux. Parmi ces maladies, celles dues à des parasites peuvent occuper une place primordiale surtout lorsqu'il n'y a pas de bon schéma prophylactique. Au parc zoologique de Hann, les animaux font souvent l'objet de traitements antiparasitaires mais la fréquence de ces traitements pourrait être mieux adaptée si les données d'infestations parasitaires y étaient mieux connues. Ainsi l'objectif de cette étude était d'étudier l'infestation par les parasites chez une partie des animaux de ce zoo.

1. Ecole inter-Etats des sciences et médecine vétérinaires, BP 5077 Fann, Dakar, Sénégal.

* Auteur pour la correspondance

Tél. : +221338651008; email : d.dahourou@eismv.org

\section{MATERIEL ET METHODES}

\section{Site d'étude}

L'étude a été réalisée dans le parc zoologique de Hann, situé dans la ville de Dakar, capitale du Sénégal (figure 1). Ce zoo existe depuis 1935 et s'étend sur sept hectares, dont seulement quatre sont aménagés. Il abrite une centaine d'espèces animales comprenant des oiseaux, des reptiles, des mammifères, dont quelques espèces emblématiques (lions, pumas, tigres, chimpanzés et buffles). Les animaux sont détenus dans des enclos, des cages, des fosses, ou des volières. Parmi l'ensemble des animaux concernés par cette étude, seul un lion provenait du parc national du Niokolo Koba (Sénégal) ; au moment du prélèvement il avait déjà passé plus de 10 ans au parc zoologique de Hann. Les autres animaux y étaient nés.

\section{Echantillonnage et prélèvements}

L'échantillonnage a porté sur l'ensemble des carnivores et des primates. Des matières fécales de chaque animal présent dans les enclos ont été collectées. Des échantillons ont ainsi été obtenus pour huit lions (Panthera leo leo), deux hyènes tachetées (Crocuta crocuta), un tigre (Panthera tigris), deux pumas (Puma concolor), un chacal (Canis aureus), quatre babouins (Papio anubis), un singe rouge (Erythrocebus patas) et cinq chimpanzés (Pan troglodytes). Les prélèvements ont été réalisés pendant le mois d'août 2016. Un pot à prélèvement a été utilisé pour récolter les matières fécales de chaque individu. Les animaux ont été identifiés morphologiquement et observés tous 


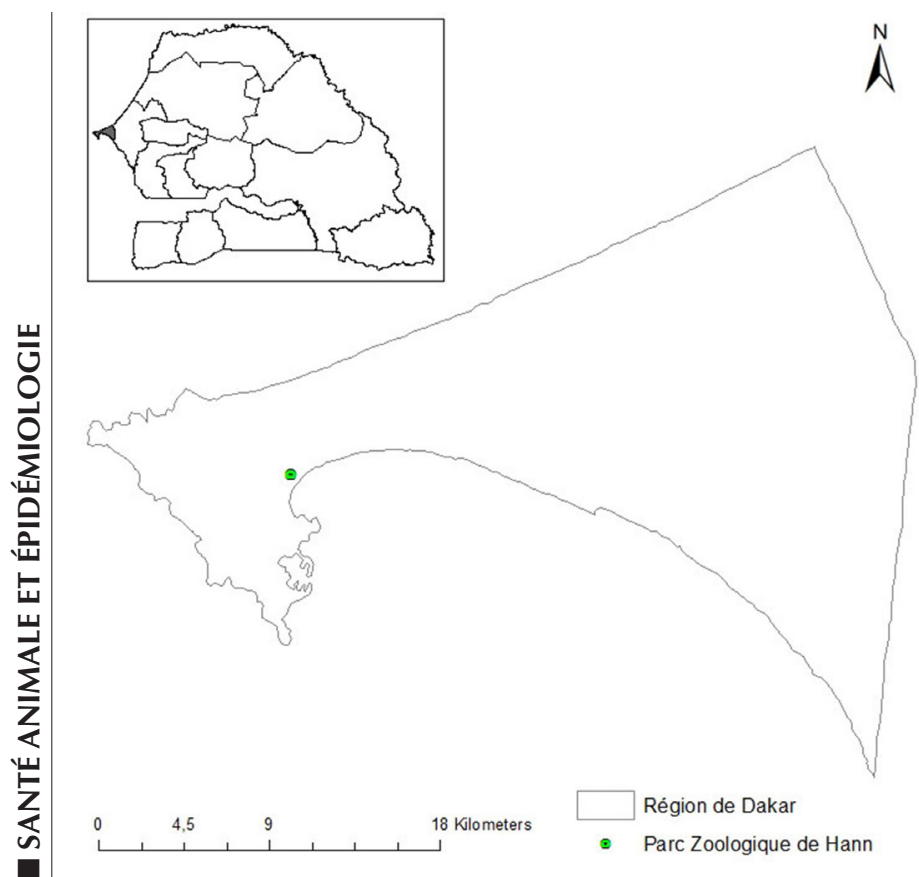

Figure 1 : localisation du parc zoologique de Hann à Dakar au Sénégal.

les jours pendant l'étude. Chaque fois qu'un individu déféquait, ses matières fécales étaient aussitôt récupérées à l'aide d'une spatule fixée sur un long manche. Les échantillons recueillis ont été conservés dans du formol à $10 \%$ et transportés au Laboratoire de parasitologie et mycologie de l'Ecole inter-Etats des sciences et médecine vétérinaires (Eismv) de Dakar pour les analyses coprologiques.

\section{Analyses parasitologiques}

Au laboratoire, deux techniques ont été utilisées pour évaluer l'infestation parasitaire : la technique qualitative de Telemann-Rivas, utilisant des solutions d'acide acétique à $5 \%$ et d'éther, et la technique quantitative en cellules de Mac Master, pour laquelle une solution saturée de chlorure de sodium a été employée. Ces deux techniques ont été appliquées selon Beugnet et al. (2006). L'identification des formes parasitaires au microscope a été réalisée avec les clés d'identification de Beugnet et al. (2006), et de Jessee et al. (1970).

\section{Analyses statistiques}

Pour chaque espèce de parasite, la moyenne arithmétique des œufs par gramme de fèces (opg) a été calculée pour les animaux infestés.

\section{RESULTATS}

\section{Infestation globale}

Sur les 24 échantillons analysés, 16 étaient positifs pour au moins une espèce de parasites, soit une prévalence de 66,7 \%. Parmi les 16 échantillons positifs, 12 contenaient au moins deux espèces de parasites et 4 n'en contenaient qu'une seule. Tous les animaux positifs étaient infestés par au moins une espèce d'helminthes, alors que seuls quatre d'entre eux étaient infestés par des protozoaires.

\section{Infestation des carnivores}

Des œufs de Toxascaris leonina ont été retrouvés chez les huit lions, chacun d'entre eux étant par ailleurs infesté par au moins trois espèces de parasites (tableau I). Ancylostoma sp. a été identifié chez les hyènes, le chacal et le tigre, alors que des œufs de Toxocara cati ont été observés chez l'un des pumas (tableau I).

\section{Infestation des primates non humains}

Deux babouins de l'échantillon étaient excréteurs de kystes d'Entamoeba sp. Ces kystes étaient de forme sphérique avec chacun quatre nuclei et des dépôts de chromatine. Trois babouins étaient infestés par Trichuris sp. avec un niveau d'infestation moyen de 433 opg (tableau II). Aucun élément parasitaire n'a été retrouvé dans les selles du singe rouge et des chimpanzés.

\section{DISCUSSION}

Les prélèvements réalisés ont été conservés au formol car ils étaient collectés durant toute la journée, mais ils n'étaient analysés que dans un délai de 24 à 48 heures. En outre, il n'y avait pas de possibilité de conserver ces matières fécales à $+4{ }^{\circ} \mathrm{C}$ du fait de l'indisponibilité de moyens de réfrigération. Ce mode de conservation a pu entraîner une sous-estimation du nombre d'opg (Cabaret, 1981) qui

\section{Tableau I}

Parasites identifiés chez les carnivores dans le parc zoologique de Hann à Dakar au Sénégal

\begin{tabular}{|c|c|c|c|}
\hline $\begin{array}{l}\text { Hôte } \\
\text { Parasite }\end{array}$ & $\begin{array}{l}\text { Nb. d'échantillons } \\
\text { analysés }\end{array}$ & $\begin{array}{l}\text { Nb. d'animaux } \\
\text { infestés }\end{array}$ & $\begin{array}{l}\text { OPG * } \\
\text { moyen }\end{array}$ \\
\hline \multicolumn{4}{|l|}{ Lion } \\
\hline Toxascaris leonina & 8 & 8 & 681 \\
\hline Capillaria sp. & 8 & 2 & 250 \\
\hline Toxocara cati & 8 & 7 & 312 \\
\hline Strongyloides sp. & 8 & 3 & 150 \\
\hline Isospora sp. & 8 & 2 & 650 \\
\hline Ancylostoma sp. & 8 & 6 & 350 \\
\hline \multicolumn{4}{|l|}{ Hyène } \\
\hline Ancylostoma sp. & 2 & 2 & 350 \\
\hline Strongyloïdes sp. & 2 & 2 & 200 \\
\hline \multicolumn{4}{|l|}{ Tigre } \\
\hline Ancylostoma sp. & 1 & 1 & 300 \\
\hline \multicolumn{4}{|l|}{ Puma } \\
\hline Toxocara cati & 2 & 1 & 100 \\
\hline \multicolumn{4}{|l|}{ Chacal } \\
\hline Ancylostoma sp. & 1 & 1 & 300 \\
\hline
\end{tabular}

\section{Tableau II}

Infestation des primates dans le parc zoologique de Hann à Dakar au Sénégal

\begin{tabular}{lccc|}
$\begin{array}{l}\text { Hôte } \\
\text { Parasite }\end{array}$ & $\begin{array}{c}\text { Nb. d'échantillons } \\
\text { analysés }\end{array}$ & $\begin{array}{c}\text { Nb. d'animaux } \\
\text { infestés }\end{array}$ & $\begin{array}{c}\text { OPG * } \\
\text { moyen }\end{array}$ \\
\hline $\begin{array}{l}\text { Babouin } \\
\text { Entamoeba sp. }\end{array}$ & 4 & & \\
$\quad$ Trichuris sp. & 4 & 2 & - \\
\hline
\end{tabular}

\footnotetext{
${ }^{*}$ Eufs par gramme de fèces
} 
ont été trouvés faibles pour la plupart des espèces parasitaires, car l'ajout de la solution formolée a entraîné une dilution des matières fécales (Euzeby, 1981). Le choix de la technique de Teleman-Rivas s'est justifié par sa bonne sensibilité dans les coprologies réalisées avec des matières fécales grasses, comme celle des carnivores et des primates (Beugnet et al., 2004), et aussi par le fait que certaines matières fécales contenaient du mucus : la présence d'acide acétique dans la réaction permettait de dissoudre le mucus et de faciliter l'observation.

L'étude a montré que la majorité $(66,7 \%)$ des animaux examinés au parc zoologique de Hann était infestée par au moins un type de parasites. Dans d'autres jardins zoologiques à travers le monde, des prévalences également élevées de $76,6 \%$ et $49,1 \%$ ont été trouvées au Nigeria (respectivement par Opara et al., 2010, et Adeniyi et al., 2015), $68 \%$ en Inde (Mir et al., 2016), et 56,3 \% en Malaisie (Lim et al., 2008). Cette forte prévalence est à mettre en relation avec les conditions climatiques de la zone d'étude, et particulièrement la température et l'humidité, favorables au développement des stades libres infestants, d'autant que les prélèvements ont été réalisés au mois d'août, c'est-à-dire pratiquement à la fin de la saison des pluies à Dakar. En outre, les enclos des animaux ne sont pas nettoyés régulièrement ; les œufs d'helminthes et les kystes de protozoaires peuvent ainsi se développer et devenir infestants. Le déparasitage n'est pas régulier pour les animaux du parc et le dernier traitement antiparasitaire réalisé avant nos prélèvements datait de plus d'un an.

Les animaux examinés étaient porteurs de nématodes et de protozoaires à des prévalences respectives de $100 \%$ et $25 \%$ chez les animaux infestés. Aucune espèce de trématode ou de cestode n'a été identifiée. Rahman et al. (2014) au Bangladesh, Opara et al. (2010) au Nigeria, et Mir et al. (2016) en Inde font le même constat. Ceci est très probablement lié au fait que les trématodes et la majorité des cestodes, à l'exception de Hymenolepis nana, ont besoin d'hôtes intermédiaires, absents des enclos et cages, pour boucler leur cycle évolutif (Atanaskova et al., 2011)

Chez les carnivores, chez lesquels les ascarides et les ankylostomes ont été les plus observés, les lions ont été les plus infestés, suivis des hyènes. Des résultats similaires ont été trouvés en Inde par Varadharajan et Kandasamy (2000) qui ont observé que Toxocara sp. et Ancylostoma sp. étaient les espèces les plus fréquentes chez ces animaux. La forte fréquence d'infestation des lions et des hyènes vis-à-vis des ascarides pouvait être due au fait que ces animaux vivaient groupés dans les mêmes enclos, ce qui n'était pas le cas du tigre, des pumas et du chacal qui étaient dans des enclos individuels.

Chez les primates, seuls trois des babouins ont été trouvés infestés. En outre, le babouin non excréteur était isolé de ses congénères. Dans les matières fécales du singe rouge et des chimpanzés, aucun œuf n'a été retrouvé mais cela ne permet pas d'affirmer que ces animaux n'étaient pas infestés. Chez les babouins, Trichuris sp. a été l'espèce parasitaire la plus retrouvée en coproscopie. Cette observation a également été rapportée par Egbetade et al. (2014) au Nigeria. Le résultat coprologique négatif du singe rouge et des chimpanzés pouvait être dû à l'aménagement de leurs enclos. Ceux-ci étaient en effet construits de telle sorte que les singes vivaient presque tout le temps en hauteur, perchés sur des branches d'arbres, les barres des enclos ou des cordes, ayant ainsi peu de contact avec le sol, lieu de développement des formes infestantes des parasites. Les kystes d'Entamoeba sp. identifiés étaient caractérisés par la présence de quatre nuclei, laissant penser qu'il s'agissait de l'espèce Entamoeba histolytica.

Les infestations des divers groupes d'animaux pourraient s'expliquer par la contamination de leur environnement par l'eau de boisson ou les aliments, mais aussi par les soigneurs qui ont été signalés comme pouvant transporter des parasites par le biais de leurs chaussures, vêtements, mains ou par l'intermédiaire des outils de travail (Adetunji, 2014 ; Otegbade et Morenikeji, 2014). Certains parasites comme E. histolytica ont un potentiel zoonotique. Bien que le risque d'une transmission à partir de l'animal soit minimal (Thompson et Smith, 2011), la présence de ce parasite pourrait poser un problème de santé publique pour les soigneurs mais également pour les visiteurs. Dans un tel contexte, il serait important de sensibiliser les soigneurs et les visiteurs pour qu'ils évitent de manger à proximité des enclos des animaux, particulièrement si les aliments ont été en contact avec le sol ou les animaux eux-mêmes, comme cela peut arriver dans le cas des enfants.

\section{CONCLUSION}

La majorité des animaux du parc était infestée par diverses espèces de parasites. Cependant, aucun animal n'a présenté de signes cliniques en relation avec les parasites identifiés lors des prélèvements, ce qui pourrait témoigner d'infestation subclinique. Il serait intéressant de mettre en place un suivi plus régulier des infestations parasitaires, en fonction des saisons, et d'utiliser les résultats pour adapter les mesures de lutte, sanitaires ou médicales.

\section{Remerciements}

Nous tenons à remercier le personnel du parc zoologique de Hann pour l'assistance particulière lors des prélèvements. Nous remercions particulièrement le conservateur du parc pour avoir autorisé cette étude au sein du zoo.

\section{REFERENCES}

Adeniyi I.C., Morenikeji O.A., Emikpe B.O., 2015. The prevalence of gastro-intestinal parasites of carnivores in university zoological gardens in South West Nigeria. J. Vet. Med. Anim. Health, 7 (4): 135139, doi: 10.5897/JVMAH2014.0336

Adetunji V.E., 2014. Prevalence of gastro-intestinal parasites in primates and their keepers from two zoological gardens in Ibadan, Nigeria. Sokoto J. Vet. Sci., 12 (2): 25-30, doi: 10.4314/sokjvs.v12i2.5

Asres A., Amha N., 2014. Effect of stress on animal health: a review. J. Biol. Agric. Healthc., 4 (27): 116-121

Atanaskova E., Kochevski Z., Stefanovska J., Nikolovski G., 2011 Endoparasites in wild animals at the zoological garden in Skopje, Macedonia. J. Threat. Taxa, 3 (7): 1955-1958, doi 10.11609/JoTT.02440.1955-8

Beugnet F., Bourdoiseau G., Dang H., 2004. Abrégé de parasitologie clinique des carnivores domestiques, vol. 1, Parasitoses digestives. Kalianxis, Paris, France, 266 p.

Beugnet F., Polack B., Dang H., 2006. Atlas de coproscopie. Kalianxis, Paris, France, $277 \mathrm{p}$.

Cabaret J., 1981. Diagnostic quantitatif des œufs de strongles digestifs et des larves de protostrongylidés chez les ovins. Influence de la durée et du mode de conservation des fèces. Rec. Med. Vet., 157 (4) : 347-349

Egbetade A., Akinkuotu O., Jayeola O., Niniola A., Emmanuel N., Olugbogi E., Onadeko S., 2014. Gastrointestinal helminths of resident wildlife at the Federal University of Agriculture Zoological Park, Abeokuta. Sokoto J. Vet. Sci., 12 (3): 26-31, doi: 10.4314/sokjvs. v12i3.5

Euzeby J., 1981. Diagnostic expérimental des helminthoses animales (animaux domestiques, animaux de laboratoire, primates). Travaux pratiques d'helminthologie vétérinaire, tome II. Informations techniques des services vétérinaires, Paris, France, 350 p. 
Jessee M.T., Schilling P.W., Stunkard J.A., 1970. Identification of intestinal helminth eggs in old world primates. Lab. Anim. Care, 20 (1): 83-87

Lim Y.A.L., Ngui R., Shukri J., Rohela M., Mat Naim H.R., 2008. Intestinal parasites in various animals at a zoo in Malaysia. Vet. Parasitol., 157 (1-2): 154-159, doi: 10.1016/j.vetpar.2008.07.015

Mir A.Q., Dua K., Singla L.D., Sharma S., Singh M.P., 2016. Prevalence of parasitic infection in captive wild animals in Bir Moti Bagh mini zoo (Deer Park), Patiala, Punjab. Vet. World, 9 (6): 540-543, doi: 10.14202/vetworld.2016.540-543

Mooney H.A., 2010. The ecosystem-service chain and the biological diversity crisis. Philos. Trans. R. Soc. Lond. B. Biol. Sci., 365 (1537): 31 39, doi: 10.1098/rstb.2009.0223

Morgan K.N., Tromborg C.T., 2007. Sources of stress in captivity. Appl. Anim. Behav. Sci., 102 (3): 262-302, doi: 10.1016/j. applanim.2006.05.032

\section{Summary}

Dahourou L.D., Gbati O.B., Nacanabo I., Diatta C., Pangui L.J. Gastrointestinal parasitism in wildlife at Hann Zoological Park (Senegal)

Fecal samples from 24 animals of eight different species were collected at Hann Zoological Park in Senegal. They were analyzed with the Telemann-Rivas qualitative microscopic method and Mac Master quantitative method. Of all the samples, $66.7 \%$ were positive, and each positive animal was infested with at least one helminth egg species, whereas protozoa were present in only four animals. In carnivores, the eggs of parasites such as Toxascaris leonina and Toxocara cati, and hookworm eggs were the most present, whereas in primates, the eggs of Trichuris sp. and Entamoeba sp. have been identified. This study provides a basis for the establishment of treatments in these animals.

Keywords: primate, Carnivora, endoparasite, zoo animal, Dakar, Senegal
Opara M.N., Osuji C.T., Opara J.A., 2010. Gastrointestinal parasitism in captive animals at the zoological garden, Nekede Owerri, Southeast Nigeria. Rep. Opin., 2 (5): 21-28

Otegbade A.C., Morenikeji O.A., 2014. Gastrointestinal parasites of birds in zoological gardens in south-west Nigeria. Trop. Biomed., 31(1): 54-62

Rahman S.M., Dey A.R., Kundu U.K., Begum N., 2014. Investigation of gastrointestinal parasites of herbivores at Dhaka National Zoological Garden of Bangladesh. J. Bangladesh Agric. Univ., 12 (1): 79-85, doi: 10.3329/jbau.v12i1.21245

Thompson R.C.A., Smith A., 2011. Zoonotic enteric protozoa. Vet. Parasitol., 182 (1): 70-78, doi: 10.1016/j.vetpar.2011.07.016

Varadharajan A., Kandasamy A., 2000. A survey of gastro-intestinal parasites of wild animals in captivity in the V.O.C. Park and mini zoo, Coimbatore. Zoo's Print J., 15 (5): 257-258

\section{Resumen}

Dahourou L.D., Gbati O.B., Nacanabo I., Diatta C., Pangui L.J. Parasitismo gastrointestinal en la fauna silvestre del Parque Zoológico de Hann (Senegal)

Se recogieron muestras fecales de 24 animales de ocho especies diferentes en el Parque Zoológico de Hann en Senegal. Fueron analizados con el método microscópico cualitativo Telemann-Rivas y el método cuantitativo Mac Master. De todas las muestras, el $66,7 \%$ fueron positivas, y cada animal positivo fue infestado con al menos una especie de huevos de helminto, mientras que los protozoos estaban presentes en sólo cuatro animales. En carnívoros, los huevos de parásitos como Toxascaris leonina y Toxocara cati, y los huevos de anquilostomas fueron los más presentes, mientras que, en primates, los huevos de Trichuris sp. y Entamoeba sp. han sido identificados. Este estudio proporciona una base para el establecimiento de tratamientos en estos animales.

Palabras clave: primate, carnívora, endoparásito, animal de parque zoológical, Dakar, Senegal 